\title{
Lie Symmetry Analysis, Optimal Systems and Explicit Solutions of the Dispersive Long Wave Equations
}

\author{
Xiaomei Xue, Yushan Bai \\ Department of Mathematics, Inner Mongolia University of Technology, Hohhot, China \\ Email:xxm15735291294@163.com,mbaiyushan@imut.edu.cn
}

How to cite this paper: Xue, X.M. and Bai, Y.S. (2018) Lie Symmetry Analysis, Optimal Systems and Explicit Solutions of the Dispersive Long Wave Equations. Journal of Applied Mathematics and Physics, 6, 2681-2696.

https://doi.org/10.4236/jamp.2018.612222

Received: November 23, 2018

Accepted: December 26, 2018

Published: December 29, 2018

Copyright ( 2018 by author(s) and Scientific Research Publishing Inc. This work is licensed under the Creative Commons Attribution International License (CC BY 4.0).

http://creativecommons.org/licenses/by/4.0/

\section{Open Access}

\begin{abstract}
In this paper, the dispersive long wave equation is studied by Lie symmetry group theory. Firstly, the Lie symmetries of this system are calculated. Secondly, one dimensional optimal systems of Lie algebra and all the symmetry reductions are obtained. Finally, based on the power series method and the extended Tanh function method, some new explicit solutions of this system are constructed.
\end{abstract}

\section{Keywords}

Dispersive Long Wave Equations, Lie Symmetry Analysis, Optimal Systems, Power Series Method, Extended Tanh Function Method, Explicit Solutions

\section{Introduction}

In mathematical physics, many significant phenomena and dynamic processes can be represented by nonlinear partial differential equations (NLPDEs) [1] [2] [3] [4]. Therefore, it is very important to find the solution of NLPDEs. A wealth of effective methods have been developed to explore the solutions of the NLPDEs, such as Adomian decomposition method [5], Darboux and Backlund transformations [6], Hirota bilinear method [7] [8] [9], and Lie symmetry method [10] [11], etc. The Lie symmetry method for constructing explicit solutions of the NLPDEs has been regarded as one of the most widely applicable methods [12] [13] [14]. Its core idea is the invariance principle of the NLPDEs under the action of Lie point transformation group (point symmetry) [10]. In recent years, there has been a great deal of research and considerable development in the symmetry field of differential equations, in terms of the number of academic papers, books and new symbolic programs dedicated to this subject [15]-[20]. 
At present, there is no general method for solving NLPDEs. Although the symmetry method has a wide range of applications in solving methods, it still faces many difficulties and challenges to promote its development. However, the symmetry method and other methods (e.g. generalized simple equation method [21], generalized Tanh function method [22], homotopy perturbation method [23] and power series method [24], etc.) are effectively combined to reflect the complementarity of each other, which makes it possible to obtain exact solutions of some NLPDEs with physical significance, and attracts the attention and research of many scholars [25] [26] [27].

In the present paper, based on the Lie group method, we will investigate the dispersive long wave equations

$$
\left\{\begin{array}{l}
u_{t}+v_{x}+\frac{1}{2}\left(u^{2}\right)_{x}=0, \\
v_{t}+\left(u v+u+u_{x x}\right)_{x}=0,
\end{array}\right.
$$

where $u$ represents the amplitude of a surface wave, propagating along the $x$-axis with a horizontal velocity. It plays an important role in nonlinear physics [28] [29], considered as a good model for the study of bidirectional solitons in water waves. In [30] [31], Eckhaus and Boiti et al. presented the extensions of Equation (1) in higher-dimensional spaces. In [32], Zhang J F et al. discussed its some new multi-soliton solutions and travelling wave solutions using the extended homogeneous balance method, etc.

The outline of this paper is as follows: in Section 2, the Lie symmetry analysis is performed for the dispersive long wave equations; in Section 3, the optimal systems and the similarity reductions of Equation (1) are researched employing Lie group analysis in the last section; in Section 4, the exact solutions for the reduced equation are obtained by using the power series method and the extended Tanh method; and in Section 5, a brief summary is done to the full text.

\section{Lie Symmetry Analysis}

We first do some preparatory work on the concept of classical Lie symmetry of general NLPDEs. Consider the $k$ th-order scalar NLPDEs of the form

$$
f^{\alpha}\left(x, u, u_{(1)}, \cdots, u_{(k)}\right)=0, \alpha=1,2, \cdots, m,
$$

where $x=\left(x_{1}, x_{2}, \cdots, x_{n}\right)$ denotes $n$ independent variables, $u=\left(u_{1}, u_{2}, \cdots, u_{m}\right)$ denotes $m$ independent variables, and $u_{(j)}=u_{i_{1} i_{2} \cdots i_{j}}^{\alpha}\left(j=1,2, \cdots, k, i_{s}=1,2, \cdots, j\right)$ denote the partial derivatives of $u^{\alpha}$ with respect to $x_{i}=(i=1,2, \cdots, n)$ up to th-order, i.e.

$$
u_{(j)}^{\alpha}=\frac{\partial^{j} u^{\alpha}}{\partial x_{i_{1}} \partial x_{i_{2}} \cdots \partial x_{i_{j}}} .
$$

Suppose that the one-parameter Lie group of point transformations

$$
\begin{aligned}
& x_{i}^{*}=X(x, u ; \varepsilon)=x+\varepsilon \xi^{i}(x, u)+O\left(\varepsilon^{2}\right), \\
& u_{q}^{*}=U(x, u ; \varepsilon)=u+\varepsilon \eta^{\alpha}(x, u)+O\left(\varepsilon^{2}\right),
\end{aligned}
$$


where $i=1,2, \cdots, n, q=1,2, \cdots, m . \varepsilon$ is an infinitesimal parameter, $\xi^{i}, \eta^{\alpha}$ are some smooth function with variables $x, u$.

Theorem 1. [10] (The Infinitesimal Invariant Formula for NLPDEs) If

$$
X=\xi^{i} \frac{\partial}{\partial x_{i}}+\eta^{\alpha} \frac{\partial}{\partial u^{\alpha}}
$$

is the infinitesimal generator of the one-parameter Lie group of transformations for (3), and the $k$-th prolongation of the infinitesimal generator is

$$
X^{(k)}=X+\eta_{i}^{(1) \alpha} \frac{\partial}{\partial u_{i}^{\alpha}}+\cdots+\eta_{i_{1}, i_{2} \cdots i_{k}}^{(k) \alpha} \frac{\partial}{\partial u_{i_{1}, i_{2} \cdots i_{k}}^{\alpha}}
$$

where the prolongation of the infinitesimals satisfy the following recurrence relation

$$
\begin{gathered}
\eta_{i}^{(1) \alpha}=D_{i} \eta^{\alpha}-\left(D_{i} \xi^{j}\right) u_{j}^{\alpha}, i=1,2, \cdots, n, \\
\eta_{i_{1}, i_{2} \cdots i_{k}}^{(k) \alpha}=D_{i_{k}} \eta_{i_{1}, i_{2} \cdots i_{k-1}}^{(k-1) \alpha}-\left(D_{i_{k}} \xi^{j}\right) u_{i_{1}, i_{2} \cdots i_{k-1}}^{\alpha}, i_{l}=1,2, \cdots, k(k \geq 2)
\end{gathered}
$$

where $D_{i}$ denotes the total derivative operator defined as

$$
D_{i}=\frac{\partial}{\partial x_{i}}+u_{i}^{\alpha} \frac{\partial}{\partial u}+u_{i j}^{\alpha} \frac{\partial}{\partial u_{j}}+\cdots+u_{i_{1} i_{2} \cdots i_{j}}^{\alpha} \frac{\partial}{\partial u_{i_{1} i_{2} \cdots i_{j}}}+\cdots, i=1,2, \cdots, n .
$$

That one-parameter Lie group of transformations (3) is the Lie symmetry of Equation (2), if and only if

$$
\left.X^{(k)} f^{\alpha}\left(x, u, u_{(1)}, \cdots, u_{(k)}\right)\right|_{f^{\alpha}\left(x, u, u_{(1)}, \cdots, u_{(k)}\right)=0}=0 .
$$

Next, we calculate the Lie symmetry of Equation (1). With regard to the infinitesimal generator of Equation (1), it can be expressed from (4) as the following form

$$
X=\xi \frac{\partial}{\partial x}+\tau \frac{\partial}{\partial t}+\eta \frac{\partial}{\partial u}+\phi \frac{\partial}{\partial v} .
$$

Applying the Theorem 1 to Equation (1), we have

$$
\left\{\begin{array}{l}
\left.X^{(3)}\left[u_{t}+v_{x}+\frac{1}{2}\left(u^{2}\right)_{x}\right]\right|_{u_{t}+v_{x}+\frac{1}{2}\left(u^{2}\right)_{x}=0, v_{t}+\left(u v+u+u_{x x}\right)_{x}=0}=0, \\
\left.X^{(3)}\left[v_{t}+\left(u v+u+u_{x x}\right)_{x}\right]\right|_{u_{t}+v_{x}+\frac{1}{2}\left(u^{2}\right)_{x}=0, v_{t}+\left(u v+u+u_{x x}\right)_{x}=0}=0 .
\end{array}\right.
$$

By simplifying (7), we can get the following overdetermined equations about $\xi, \tau, \eta, \phi$

$$
\left\{\begin{array}{l}
\xi_{u}=\xi_{v}=0, \tau_{x}=\tau_{u}=\tau_{v}=0, \eta_{x}=\eta_{t}=\eta_{v}=0, \\
\phi_{x}=\phi_{t}=\phi_{u}=0,2 \eta_{u}+2 v \eta_{u}-\phi=0 \\
2 \xi_{x}+2 v \xi_{x}+\phi=0, \tau_{t}+v \tau_{t}+\phi=0 \\
2 \eta+2 v \eta-2 \xi_{t}-2 v \xi_{t}-u \phi=0, \phi-\phi_{v}-v \phi_{v}=0 .
\end{array}\right.
$$

From (8) it is easy to caculate that the only solution of this system is

$$
\xi=k_{4} x+k_{3} t+k_{1}, \tau=2 k_{4} t+k_{2}, \eta=-k_{4} u+k_{3}, \phi=-2 k_{4} v-2 k_{4},
$$


where $k_{1}, k_{2}$ and $k_{3}$ are arbitrary constants. Accordingly, the symmetry groups of Equation (1) can be written as

$$
X_{1}=\frac{\partial}{\partial x}, X_{2}=\frac{\partial}{\partial t}, X_{3}=t \frac{\partial}{\partial x}+\frac{\partial}{\partial u}, X_{4}=x \frac{\partial}{\partial x}+2 t \frac{\partial}{\partial t}-u \frac{\partial}{\partial u}-2(v+1) \frac{\partial}{\partial v}
$$

The infinitesimal generators (10) correspond to a four-parameter Lie group of nontrivial point transformations acting on $(x, t, u, v)$-space.

\section{Optimal System and Symmetry Reductions}

\subsection{Optimal System}

In this section, we study how to construct the one-dimensional optimal system of Equation (1) in order to obtain more abundant group invariant solutions. The basic method of constructing it is to simplify the expression of Lie algebra by using a variety of adjoint transformations on the most general expression of Lie algebra. The adjoint transformation is expressed as the following series form

$$
\begin{aligned}
& \operatorname{Ad}\left(\exp \left(\varepsilon X_{i}\right)\right) X_{j} \\
& =X_{j}-\varepsilon\left[X_{i}, X_{j}\right]+\frac{\varepsilon^{2}}{2}\left[X_{i},\left[X_{i}, X_{j}\right]\right]-\frac{\varepsilon^{3}}{3 !}\left[X_{i},\left[X_{i},\left[X_{i}, X_{j}\right]\right]\right]+\cdots,
\end{aligned}
$$

where $\varepsilon$ is a parameter, and $\left[X_{i}, X_{j}\right]$ is the usual commutator, given by

$$
\left[X_{i}, X_{j}\right]=X_{i} X_{j}-X_{j} X_{i} \text {. }
$$

Hence we can get the following commutator Table 1 and the adjoint representation Table 2.

According to the method of constructing one dimensional optimal system in [11], we set up the following non-zero vector field with arbitrary coefficients $a_{1}, a_{2}, a_{3}$ and $a_{4}$, which is a Lie algebras made up of (10)

$$
X=a_{1} X_{1}+a_{2} X_{2}+a_{3} X_{3}+a_{4} X_{4},
$$

and simplify the coefficients of the vector as much as possible. Without loss of generality, suppose first that $a_{4} \neq 0$ and set up $a_{4}=1$, then the vector $X$ becomes $X=a_{1} X_{1}+a_{2} X_{2}+a_{3} X_{3}+X_{4}$ To eliminate the coefficient of $X_{1}$, we use $X_{1}$ to act on $X$ by means of the adjoint operation, i.e.

$$
X^{\prime}=\operatorname{Ad}\left(\exp \left(\varepsilon_{1} X_{1}\right)\right) X=a_{2} X_{2}+a_{3} X_{3}+X_{4},
$$

where the group parameter $\varepsilon_{1}=a_{1}$. Then continue to eliminate $X_{2}, X_{3}$ by using one after another $X_{2}, X_{3}$ to act on $X^{\prime}$, the vector becomes

$$
X^{\prime \prime}=A d\left(\exp \left(\varepsilon_{3} X_{3}\right)\right) A d\left(\exp \left(\varepsilon_{2} X_{2}\right)\right) X^{\prime}=X_{4},
$$

where the group parameters $\varepsilon_{2}=a_{2} / 2, \varepsilon_{3}=-a_{3}$. It can be seen easily that the vector form can not be simplified much more. Secondly, suppose that $a_{4}=0, a_{3} \neq 0$ and set up $a_{3}=1$, the vector $X$ becomes $X=a_{1} X_{1}+a_{2} X_{2}+X_{3}$. To eliminate the coefficient of the vector $X_{1}$ we use $X_{2}$ to act on $X$ by means of the adjoint operation, i.e.

$$
X^{\prime \prime \prime}=\operatorname{Ad}\left(\exp \left(\varepsilon_{4} X_{2}\right)\right) X=a_{2} X_{2}+X_{3},
$$


Table 1. Commutator table.

\begin{tabular}{ccccc}
\hline$\left[X_{i}, X_{j}\right]$ & $X_{1}$ & $X_{2}$ & $X_{3}$ & $X_{4}$ \\
\hline$X_{1}$ & 0 & 0 & 0 & $X_{1}$ \\
$X_{2}$ & 0 & 0 & $X_{1}$ & $2 X_{2}$ \\
$X_{3}$ & 0 & $-X_{1}$ & 0 & $-X_{3}$ \\
$X_{4}$ & $-X_{1}$ & $-2 X_{2}$ & $X_{3}$ & 0 \\
\hline
\end{tabular}

Table 2. Adjoint representation table.

\begin{tabular}{ccccc}
\hline$A d$ & $X_{1}$ & $X_{2}$ & $X_{3}$ & $X_{4}$ \\
\hline$X_{1}$ & $X_{1}$ & $X_{2}$ & $X_{3}$ & $X_{4}-\varepsilon X_{1}$ \\
$X_{2}$ & $X_{1}$ & $X_{2}$ & $X_{3}-\varepsilon X_{1}$ & $X_{4}-2 \varepsilon X_{2}$ \\
$X_{3}$ & $X_{1}$ & $X_{2}+\varepsilon X_{1}$ & $X_{3}$ & $X_{4}+\varepsilon X_{3}$ \\
$X_{4}$ & $X_{1} \mathrm{e}^{\varepsilon}$ & $X_{2} \mathrm{e}^{2 \varepsilon}$ & $X_{3} \mathrm{e}^{-\varepsilon}$ & $X_{4}$ \\
\hline
\end{tabular}

where the group parameter $\varepsilon_{4}=a_{1}$. Obviously, it can not continue to simplify by using adjoint operators. Thirdly, suppose that $a_{4}=0, a_{3}=0, a_{2} \neq 0$ and set up $a_{2}=1$, the vector is already the simplest form as $X=a_{1} X_{1}+X_{2}$. Last suppose that $a_{4}=0, a_{3}=0, a_{2} \neq 0, a_{1} \neq 0$ and set up $a_{1}=1$, that can only be $X=X_{1}$.

To summarize, we state the result that the one-dimensional optimal system of symmetry groups (10) is

$$
\left\{X_{1}, X_{2}, X_{3}, X_{4}, a X_{1}+X_{2}, a X_{2}+X_{3}\right\}
$$

where $a$ is arbitrary constant.

\subsection{Symmetry Reductions}

In the present section, we present all possible similarity reduction forms of Equation (1), which is an indispensable step to solve the NLPDEs by the symmetry method.

For the symmetry $X_{4}$, the corresponding characteristic equation is

$$
\frac{\mathrm{d} x}{x}=\frac{\mathrm{d} t}{2 t}=\frac{\mathrm{d} u}{-u}=\frac{\mathrm{d} v}{-2(v+1)},
$$

hence we can get a similarity independent variable from (11) defined as $\varsigma=x t^{-1 / 2}$ and group invariant solutions defined as

$$
u(x, t)=t^{-1 / 2} F(\varsigma), v(x, t)=-1+t^{-1} H(\varsigma),
$$

which satisfy the following reduced equation

$$
\left\{\begin{array}{l}
F+\varsigma F^{\prime}-2 F F^{\prime}-2 H^{\prime}=0 \\
2 H-2 H F^{\prime}+\varsigma H^{\prime}-2 F H^{\prime}-2 F^{(3)}=0
\end{array}\right.
$$

where $F^{\prime}=\frac{\mathrm{d} F}{\mathrm{~d} \varsigma}, H^{\prime}=\frac{\mathrm{d} H}{\mathrm{~d} \varsigma}$.

For other symmetries in the optimal system, the reduction method is the same as $X_{4}$. The results are shown in Table 3 . 


\section{Explicit Solution of the Dispersive Long Wave Equation}

In the third section, we obtain the one-dimensional optimal system of Equation (1), and give the reduction equation corresponding to each symmetry in the optimal system in Table 3. The reduction equations corresponding to $X_{1}$ and $X_{3}$ can be easily solved by Mathematica, where the process is omitted. For other symmetries in the optimal system, it is very difficult to get directly through the calculation software. In this connection, we will use two methods to solve the rest of reduction equations, namely, the power series method and the extended Tanh function method.

\subsection{Explicit Power Series Solutions of the Reduction Equation (A)}

The power series method is a useful approach to solve higher order ordinary differential equations. A large number of solutions for ordinary differential equations can be constructed by utilizing the method.

Suppose that the power series solution is the following form

$$
F(\varsigma)=\sum_{n=0}^{\infty} c_{n} \varsigma^{n}, H(\varsigma)=\sum_{n=0}^{\infty} s_{n} \varsigma^{n},
$$

where $c_{n}, s_{n}$ is undetermined coefficient. Substituting (12) into (A), we get

$$
\left\{\begin{array}{l}
\sum_{n=1}^{\infty} c_{n} \varsigma^{n}+c_{0}+\sum_{n=1}^{\infty} n c_{n} \varsigma^{n}-2 \sum_{n=1}^{\infty} \sum_{k=0}^{n}(n+1-k) c_{k} c_{n+1-k} \varsigma^{n} \\
\quad-2 c_{0} c_{1}-2 \sum_{n=1}^{\infty}(n+1) s_{n+1} \varsigma^{n}-2 s_{1}=0 \\
2 \sum_{n=1}^{\infty} s_{n} \varsigma^{n}+2 s_{0}-2 \sum_{n=1}^{\infty} \sum_{k=0}^{n}(n+1-k) s_{k} c_{n+1-k} \varsigma^{n}-2 s_{0} c_{1} \\
\quad+\sum_{n=1}^{\infty} n s_{n} \varsigma^{n}-2 \sum_{n=1}^{\infty} \sum_{k=0}^{n}(n+1-k) c_{k} s_{n+1-k} \varsigma^{n}-2 c_{0} s_{1} \\
\quad-2 \sum_{n=1}^{\infty}(n+1)(n+2)(n+3) c_{n+3} \varsigma^{n}-12 c_{3}=0 .
\end{array}\right.
$$

Through comparing the coefficients of $\varsigma$, we can easily get the following results when $n=0$,

$$
s_{1}=\frac{c_{0}-2 c_{0} c_{1}}{2}, c_{3}=\frac{s_{0}-s_{0} c_{1}-c_{0} s_{1}}{6},
$$

when $n \geq 1$,

$$
\begin{aligned}
s_{n+1}= & \frac{1}{2(n+1)}\left[(n+1) c_{n}-2 \sum_{k=0}^{n}(n+1-k) c_{k} c_{n+1-k}\right], \\
c_{n+3}= & \frac{1}{2(n+1)(n+2)(n+3)}\left[(2+n) s_{n}-2 \sum_{k=0}^{n}(n+1-k) s_{k} c_{n+1-k}\right. \\
& \left.-2 \sum_{k=0}^{n}(n+1-k) c_{k} s_{n+1-k}\right] .
\end{aligned}
$$

The sequence $\left\{s_{n}\right\}_{1}^{\infty},\left\{c_{n}\right\}_{3}^{\infty}$ can be uniquely determined by (13) and (14) and depend on the other undetermined coefficients $s_{0}, c_{i}(i=0,1,2)$. It is easy to 
Table 3. Reduction of the nonlinear long wave equation.

\begin{tabular}{|c|c|c|}
\hline $\begin{array}{l}\text { Infinitesimal } \\
\text { generator }\end{array}$ & Similarity variables & Reduction equation \\
\hline$X_{1}$ & $\begin{array}{c}\varsigma=t, \\
u(x, t)=F(\varsigma), v(x, t)=H(\varsigma) .\end{array}$ & $\begin{array}{l}F^{\prime \prime}=0 \\
H^{\prime \prime}=0\end{array}$ \\
\hline$X_{2}$ & $\begin{array}{c}\varsigma=x, \\
u(x, t)=F(\varsigma), v(x, t)=H(\varsigma) .\end{array}$ & $\begin{array}{c}F F^{\prime}+H^{\prime}=0, \\
F^{\prime}+H F^{\prime}+F H^{\prime}+F^{(3)}=0 . \quad(\mathrm{D})\end{array}$ \\
\hline$X_{3}$ & $\begin{array}{c}\varsigma=t, \\
u(x, t)=x / t+F(\varsigma), v(x, t)=H(\varsigma) .\end{array}$ & $\begin{array}{c}F+\varsigma F^{\prime}=0 \\
H+\varsigma H^{\prime}+1=0\end{array}$ \\
\hline$X_{4}$ & $\begin{array}{c}\varsigma=x t^{-1 / 2} \\
u(x, t)=t^{-1 / 2} F(\varsigma), v(x, t)=-1+t^{-1} H(\varsigma) .\end{array}$ & $\begin{array}{c}F+\varsigma F^{\prime}-2 F F^{\prime}-2 H^{\prime}=0, \\
2 H-2 H F^{\prime}+\varsigma H^{\prime}-2 F H^{\prime}-2 F^{(3)}=0 . \quad(\mathrm{A})\end{array}$ \\
\hline$X_{1}+X_{2}$ & $\begin{array}{c}\varsigma=x-t \\
u(x, t)=F(\varsigma), v(x, t)=H(\varsigma) .\end{array}$ & $\begin{array}{c}-F^{\prime}+F F^{\prime}+H^{\prime}=0 \\
F^{\prime}+H F^{\prime}-H^{\prime}+F H^{\prime}+F^{(3)}=0\end{array}$ \\
\hline$X_{2}+X_{3}$ & $\begin{array}{c}\varsigma=-t^{2}+2 x / 2, \\
u(x, t)=t+F(\varsigma), v(x, t)=H(\varsigma) .\end{array}$ & $\begin{array}{c}1+F F^{\prime}+H^{\prime}=0, \\
F^{\prime}+H F^{\prime}+F H^{\prime}+F^{(3)}=0 . \quad(\mathrm{C})\end{array}$ \\
\hline
\end{tabular}

prove that the power series solution is convergent by references [13], so the reduction Equation (A) has the following power series solution

$$
\begin{aligned}
F(\varsigma)= & c_{0}+c_{1} \varsigma+c_{2} \varsigma^{2}+c_{3} \varsigma^{3}+\sum_{n=1}^{\infty} c_{n+3} \varsigma^{n+3} \\
= & c_{0}+c_{1} \varsigma+c_{2} \varsigma^{2}+\frac{s_{0}-s_{0} c_{1}-c_{0} s_{1}}{6} \varsigma^{3} \\
& +\frac{1}{2(n+1)(n+2)(n+3)} \sum_{n=1}^{\infty}\left(2 s_{n}+n s_{n}-2 \sum_{k=0}^{n}(n+1-k) s_{k} c_{n+1-k}\right. \\
& \left.-2 \sum_{k=0}^{n}(n+1-k) c_{k} s_{n+1-k}\right) \varsigma^{n+3}, \\
H(\varsigma)=s_{0}+ & s_{1} \varsigma+\sum_{n=1}^{\infty} s_{n+1} \varsigma^{n+1} \\
= & s_{0}+\frac{c_{0}-2 c_{0} c_{1}}{2} \varsigma+\frac{1}{2(n+1)} \sum_{n=1}^{\infty}\left(c_{n}+n c_{n}-2 \sum_{k=0}^{n}(n+1-k) c_{k} c_{n+1-k}\right) \varsigma^{n+1}
\end{aligned}
$$

And then we get the following power series solution of Equation (1)

$$
\begin{aligned}
& u(x, t)= t^{-1 / 2}\left[c_{0}+c_{1}\left(x t^{-1 / 2}\right)+c_{2}\left(x t^{-1 / 2}\right)^{2}+\frac{s_{0}-s_{0} c_{1}-c_{0} s_{1}}{6}\left(x t^{-1 / 2}\right)^{3}\right. \\
&+\frac{1}{2(n+1)(n+2)(n+3)} \sum_{n=1}^{\infty}\left(2 s_{n}+n s_{n}-2 \sum_{k=0}^{n}(n+1-k) s_{k} c_{n+1-k}\right. \\
&\left.\left.-2 \sum_{k=0}^{n}(n+1-k) c_{k} s_{n+1-k}\right)\left(x t^{-1 / 2}\right)^{n+3}\right] \\
& v(x, t)=-1+t^{-1}\left[s_{0}+\frac{c_{0}-2 c_{0} c_{1}}{2}\left(x t^{-1 / 2}\right)\right. \\
&\left.+\frac{1}{2(n+1)} \sum_{n=1}^{\infty}\left((n+1) c_{n}-2 \sum_{k=0}^{n}(n+1-k) c_{k} c_{n+1-k}\right)\left(x t^{-1 / 2}\right)^{n+1}\right]
\end{aligned}
$$

where $s_{0}, c_{n}(n=0,1,2)$ are arbitrary constant. 


\subsection{Explicit Solutions of the Reduction Equation (B) Using Extended Tanh Function Method}

The extended Tanh function method is a very effective method for solving some nonlinear evolution equations proposed in recent years [33]. The method is based on the Tanh function expansion method and using the general Riccati equation as an auxiliary equation. It can transform the solution of complex equations into the solution of nonlinear algebraic equations by traveling wave transformation. Next we use this method to find the traveling wave solutions of the reduction Equation (B) in Table 3.

Suppose that the solution of the reduction Equation (B) can be expressed as the form

$$
\left\{\begin{array}{l}
F(\varsigma)=a_{0}+\sum_{i=1}^{m}\left(a_{i} \phi^{i}(\varsigma)+A_{i} \phi^{-i}(\varsigma)\right), \\
H(\varsigma)=b_{0}+\sum_{j=1}^{n}\left(b_{j} \phi^{j}(\varsigma)+B_{j} \phi^{-j}(\varsigma)\right),
\end{array}\right.
$$

where $a_{0}, b_{0}, a_{i}, A_{i}(i=1, \cdots, m), b_{j}, B_{j}(j=0,1, \cdots, n)$ are undetermined constants, and function $\phi=\phi(\varsigma)$ satisfies

$$
\phi^{\prime}=\lambda+\rho \phi+\omega \phi^{2},
$$

where $\lambda, \rho, \omega$ are arbitrary constant. By solving Equation (16), we can know that the solution of function $\phi$ can be divided into 4 categories, and amount to 27 solutions [22].

1) when $\rho^{2}-4 \lambda \omega>0$ and $\rho \omega \neq 0$ (or $\lambda \omega \neq 0$ ),

$$
\begin{gathered}
\phi_{1}=-\frac{1}{2 \omega}\left(\rho+\sqrt{\theta} \tanh \left[\frac{\sqrt{\theta}}{2} \varsigma\right]\right), \\
\phi_{2}=-\frac{1}{2 \omega}(\rho+\sqrt{\theta}(\tanh [\sqrt{\theta} \varsigma] \pm i \operatorname{sech}[\sqrt{\theta} \varsigma])) \\
\phi_{3}=-\frac{1}{2 \omega}\left(\rho+\sqrt{\theta} \operatorname{coth}\left[\frac{\sqrt{\theta}}{2} \varsigma\right]\right) \\
\phi_{4}=-\frac{1}{2 \omega}(\rho+\sqrt{\theta}(\operatorname{coth}[\sqrt{\theta} \varsigma] \pm i \operatorname{csch}[\sqrt{\theta} \varsigma])) \\
\phi_{5}=-\frac{1}{4 \omega}\left[2 \rho+\sqrt{\theta}\left(\tanh \left[\frac{\sqrt{\theta}}{4} \varsigma\right]+\operatorname{coth}\left[\frac{\sqrt{\theta}}{4} \varsigma\right]\right),\right. \\
\phi_{6}=\frac{1}{2 \omega}\left(-\rho+\frac{\sqrt{\left(B^{2}-A^{2}\right)(\theta)}-A \sqrt{\theta} \cosh [\sqrt{\theta} \varsigma]}{A \sinh [\sqrt{\theta} \varsigma]+B}\right), \\
\phi_{7}=\frac{1}{2 \omega}\left(-\rho+\frac{\sqrt{\left(B^{2}-A^{2}\right)(\theta)}+A \sqrt{\theta} \sinh [\sqrt{\theta} \varsigma]}{A \cosh [\sqrt{\theta} \varsigma]+B}\right),
\end{gathered}
$$

where $A, B$ are two nonzero constants, and satisfy $B^{2}-A^{2}>0$. 


$$
\begin{gathered}
\phi_{8}=\frac{2 \lambda \cosh [\sqrt{\theta} \varsigma / 2]}{\sqrt{\theta} \sinh [\sqrt{\theta} \varsigma / 2]-\rho \cosh [\sqrt{\theta} \varsigma / 2]}, \\
\phi_{9}=\frac{-2 \lambda \sinh [\sqrt{\theta} \varsigma / 2]}{\rho \sinh [\sqrt{\theta} \varsigma / 2]-\sqrt{\theta} \cosh [\sqrt{\theta} \varsigma / 2]}, \\
\phi_{10}=\frac{2 \lambda \cosh [\sqrt{\theta} \varsigma]}{\sqrt{\theta} \sinh [\sqrt{\theta} \varsigma]-\rho \cosh [\sqrt{\theta} \varsigma] \pm i \sqrt{\theta}}, \\
\phi_{11}=\frac{2 \lambda \sinh [\sqrt{\theta} \varsigma]}{-\rho \sinh [\sqrt{\theta} \varsigma]+\sqrt{\theta} \cosh [\sqrt{\theta} \varsigma] \pm \sqrt{\theta}}, \\
\phi_{12}=\frac{4 \lambda \sinh [\sqrt{\theta} \varsigma / 4] \cosh [\sqrt{\theta} \varsigma / 4]}{-2 \rho \sinh [\sqrt{\theta} \varsigma / 4] \cosh [\sqrt{\theta} \varsigma / 4]+2 \sqrt{\theta} \cosh ^{2}[\sqrt{\theta} \varsigma / 4]-\sqrt{\theta}} .
\end{gathered}
$$

2) when $\rho^{2}-4 \lambda \omega<0$ and $\rho \omega \neq 0$ (or $\lambda \omega \neq 0$ ),

$$
\begin{aligned}
& \phi_{13}=\frac{1}{2 \omega}\left(-\rho+\sqrt{-\theta} \tan \left[\frac{\sqrt{-\theta}}{2} \varsigma\right]\right), \\
& \phi_{14}=\frac{1}{2 \omega}[-\rho+\sqrt{-\theta}(\tan [\sqrt{-\theta} \varsigma] \pm \sec [\sqrt{-\theta} \varsigma])], \\
& \phi_{15}=-\frac{1}{2 \omega}\left(\rho+\sqrt{-\theta} \cot \left[\frac{\sqrt{-\theta}}{2} \varsigma\right]\right) \text {, } \\
& \phi_{16}=-\frac{1}{2 \omega}(\rho+\sqrt{-\theta}(\cot [\sqrt{-\theta} \varsigma] \pm \csc [\sqrt{-\theta} \varsigma])) \text {, } \\
& \phi_{17}=\frac{1}{4 \omega}\left[-2 \rho+\sqrt{-\theta}\left(\tan \left[\frac{\sqrt{-\theta}}{4} \varsigma\right]-\cot \left[\frac{\sqrt{-\theta}}{4} \varsigma\right]\right),\right. \\
& \phi_{18}=\frac{1}{2 \omega}\left(-\rho+\frac{ \pm \sqrt{\left(A^{2}-B^{2}\right)(-\theta)}-A \sqrt{-\theta} \cos [\sqrt{-\theta} \varsigma]}{A \sinh [\sqrt{-\theta} \varsigma]+B}\right) \text {, } \\
& \phi_{19}=\frac{1}{2 \omega}\left(-\rho+\frac{\sqrt{\left(A^{2}-B^{2}\right)(-\theta)}+A \sqrt{-\theta} \sinh [\sqrt{-\theta} \varsigma]}{A \cosh [\sqrt{-\theta} \varsigma]+B}\right) \text {, }
\end{aligned}
$$

where $A, B$ are two nonzero constants, and satisfy $A^{2}-B^{2}>0$.

$$
\begin{aligned}
& \phi_{20}=\frac{2 \lambda \cos [\sqrt{-\theta} \varsigma / 2]}{\sqrt{-\theta} \sin [\sqrt{-\theta} \varsigma / 2]+\rho \cos [\sqrt{-\theta} \varsigma / 2]}, \\
& \phi_{21}=\frac{-2 \lambda \sin [\sqrt{-\theta} \varsigma / 2]}{-\rho \sin [\sqrt{-\theta} \varsigma / 2]-\sqrt{-\theta} \cos [\sqrt{-\theta} \varsigma / 2]}, \\
& \phi_{22}=\frac{2 \lambda \cos [\sqrt{-\theta} \varsigma]}{\sqrt{-\theta} \sin [\sqrt{-\theta} \varsigma]+\rho \cos [\sqrt{-\theta} \varsigma] \pm \sqrt{-\theta}},
\end{aligned}
$$




$$
\begin{gathered}
\phi_{23}=\frac{2 \lambda \sin [\sqrt{-\theta} \varsigma]}{-\rho \sin [\sqrt{-\theta} \varsigma]+\sqrt{-\theta} \cosh [\sqrt{-\theta} \varsigma] \pm \sqrt{-\theta}}, \\
\phi_{24}=\frac{4 \lambda \sin [\sqrt{-\theta} \varsigma / 4] \cos [\sqrt{-\theta} \varsigma / 4]}{-2 \rho \sin [\sqrt{-\theta} \varsigma / 4] \cos [\sqrt{-\theta} \varsigma / 4]+2 \sqrt{-\theta} \cos ^{2}[\sqrt{-\theta} \varsigma / 4]-\sqrt{-\theta}} .
\end{gathered}
$$

Above formula $\phi_{1} \sim \phi_{24}$, the symbol $\theta$ is expressed as $\theta=\rho^{2}-4 \lambda \omega$.

3) when $\lambda=0$ and $\rho \omega \neq 0$,

$$
\begin{aligned}
\phi_{25} & =\frac{-\rho b}{\omega(b+\cosh [\rho \varsigma]-\sinh [\rho \varsigma])}, \\
\phi_{26} & =-\frac{\rho(\cosh [\rho \varsigma]+\sinh [\rho \varsigma])}{\omega(b+\cosh [\rho \varsigma]-\sinh [\rho \varsigma])},
\end{aligned}
$$

where $b$ is a arbitrary constant.

4) when $\omega \neq 0$ and $\lambda=\rho=0$,

$$
\phi_{27}=\frac{1}{\omega \varsigma+c},
$$

where $c$ is a arbitrary constant, and $\varsigma=x-t$.

Considering the homogeneous equilibrium between the highest order linear term and the nonlinear term in the reduction Equation (B), we can obtain $m=1, n=2$. As a result, the trial Equations (16) reduces to

$$
\left\{\begin{array}{l}
F(\varsigma)=a_{0}+a_{1} \phi(\varsigma)+\frac{A_{1}}{\phi(\varsigma)}, \\
H(\varsigma)=b_{0}+b_{1} \phi(\varsigma)+b_{2} \phi^{2}(\varsigma)+\frac{B_{1}}{\phi(\varsigma)}+\frac{B_{2}}{\phi^{2}(\varsigma)} .
\end{array}\right.
$$

In order to determine the values of undetermined coefficients $a_{0}, a_{1}, b_{0}, b_{1}, b_{2}$, substituting (16) and (17) into the reduction Equation (B) and merging the polynomial of the same power of $\phi$, and setting up each polynomial coefficient to zero, we can get the following nonlinear algebraic equations

$$
\left\{\begin{array}{c}
\phi^{0}:-\lambda A_{1}^{2}-2 \lambda B_{2}=0,-6 \lambda^{3} A_{1}-3 \lambda A_{1} B_{2}=0 \\
\phi^{1}:-\lambda A_{1}-\lambda a_{0} A_{1}-\rho A_{1}^{2}-\lambda B_{1}-2 \rho B_{2}=0, \\
\quad-12 \lambda^{2} \rho A_{1}-2 \lambda A_{1} B_{1}+2 \lambda B_{2}-2 \lambda a_{0} B_{2}-3 \rho A_{1} B_{2}=0 \\
\phi^{2}: \rho A_{1}-\rho a_{0} A_{1}-\omega A_{1}^{2}-\rho B_{1}-2 \omega B_{2}=0 \\
\quad-\lambda A_{1}-7 \lambda \rho^{2} A_{1}-8 \lambda^{2} \omega A_{1}-\lambda A_{1} b_{0}+\lambda B_{1}-\lambda a_{0} B_{1} \\
\quad-2 \rho A_{1} B_{1}+2 \rho B_{2}-2 \rho a_{0} B_{2}-\lambda a_{1} B_{2}-3 \omega A_{1} B_{2}=0 \\
\quad \vdots \\
\phi^{6}: \omega a_{1}^{2}+2 \omega b_{2}=0, \quad \\
\quad \omega a_{1}+7 \rho^{2} \omega a_{1}+8 \lambda \omega^{2} a_{1}+\omega a_{1} b_{0}-\omega b_{1}+\omega a_{0} b_{1}+2 \rho a_{1} b_{1} \\
\quad-2 \rho b_{2}+2 \rho a_{0} b_{2}+3 \lambda a_{1} b_{2}+\omega A_{1} b_{2}=0 \\
\phi^{7}: 0=0,12 \rho \omega^{2} a_{1}+2 \omega a_{1} b_{1}-2 \omega b_{2}+2 \omega a_{0} b_{2}+3 \rho a_{1} b_{2}=0 \\
\phi^{8}: 0=0,6 \omega^{3} a_{1}+3 \omega a_{1} b_{2}=0
\end{array}\right.
$$


By solving the above system with the help of Mathematic, we can get the following results

$$
\begin{aligned}
& a_{0}=1 \pm \rho, a_{1}= \pm 2 \omega, A_{1}= \pm 2 \lambda, \\
& b_{0}=-1, b_{1}=-2 \rho \omega, b_{2}=-2 \omega^{2}, B_{1}=-2 \lambda \rho, B_{2}=-2 \lambda^{2} .
\end{aligned}
$$

Now, substituting (18) into (19), we obtain explicit solutions of Equation (1) as follow.

$$
\left\{\begin{array}{l}
u_{k}(x, t)=1 \pm \rho \pm 2 \omega \phi_{k}(\varsigma) \pm \frac{2 \lambda}{\phi_{k}(\varsigma)}, \\
v_{k}(x, t)=-1-2 \rho \omega \phi_{k}(\varsigma)-2 \omega^{2} \phi_{k}^{2}(\varsigma)-\frac{2 \lambda \rho}{\phi_{k}(\varsigma)}-\frac{2 \lambda^{2}}{\phi_{k}^{2}(\varsigma)},
\end{array}\right.
$$

where $k=1,2, \cdots, 27, \varsigma=x-t$, and selecting any hyperbolic function in $\phi_{1} \sim \phi_{27}$, for example,

$$
\phi(\varsigma)=\frac{1}{2 \omega}\left(-\rho+\sqrt{-\theta} \tan \left[\frac{\sqrt{-\theta}}{2} \varsigma\right]\right) .
$$

The explicit solutions (19) become as

$$
\left\{\begin{array}{l}
u(x, t)=1 \pm \sqrt{-\theta} \tan \left[\frac{\sqrt{-\theta} \varsigma}{2}\right] \mp \frac{4 \lambda \omega}{\rho-\sqrt{-\theta} \tan \left[\frac{\sqrt{-\theta} \varsigma}{2}\right]} \\
v(x, t)=-1+\frac{\rho^{2}}{2}+\frac{1}{2} \theta \tan ^{2}\left[\frac{\sqrt{-\theta} \varsigma}{2}\right]-\frac{8 \lambda^{2} \omega^{2}}{\left(-\rho+\sqrt{-\theta} \tan \left[\frac{\sqrt{-\theta} \varsigma}{2}\right]\right)^{2}} \\
-\frac{4 \lambda \rho \omega}{-\rho+\sqrt{-\theta} \tan \left[\frac{\sqrt{-\theta} \varsigma}{2}\right]},
\end{array}\right.
$$

where $\varsigma=x-t, \theta=\rho^{2}-4 \lambda \omega<0 \quad$ (see Figure 1).

\subsection{Explicit Power Series Solutions of the Reduction Equation (C)}

In this section, we study the power series solution of the reduction Equation (C)

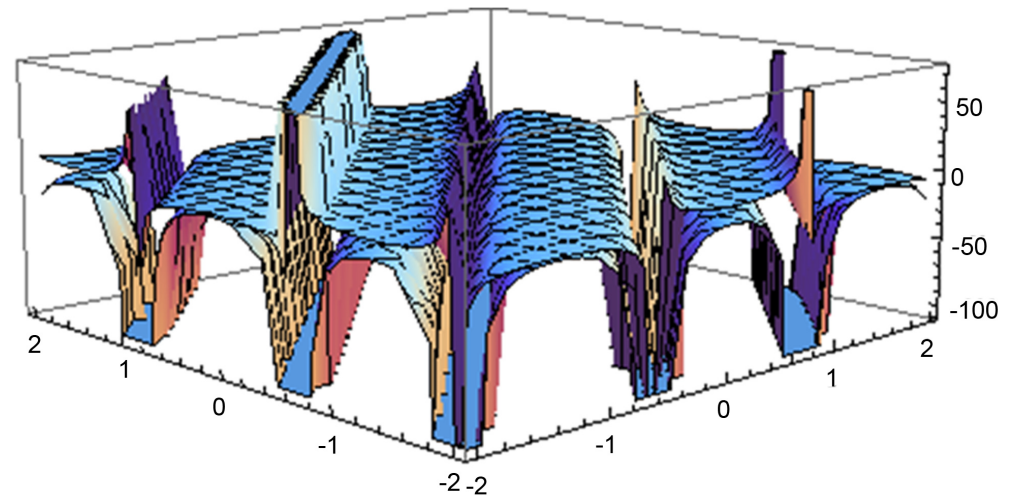

Figure 1. Plot of explicit solution above with $\lambda=4, \rho=-0.2, \omega=0.3$. 
in the form of (12). Substituting (12) into the reduction Equation (C), we get

$$
\left\{\begin{array}{l}
1+\sum_{n=1}^{\infty} \sum_{k=0}^{n}(n+1-k) c_{k} c_{n+1-k} \varsigma^{n}+c_{0} c_{1}+\sum_{n=1}^{\infty}(n+1) s_{n+1} \varsigma^{n}+s_{1}=0, \\
\sum_{n=1}^{\infty}(n+1) c_{n+1} \varsigma^{n}+c_{1}+\sum_{n=1}^{\infty} \sum_{k=0}^{n}(n+1-k) s_{k} c_{n+1-k} \varsigma^{n} \\
\quad+s_{0} c_{1}+\sum_{n=1}^{\infty} \sum_{k=0}^{n}(n+1-k) c_{k} s_{n+1-k} \varsigma^{n}+c_{0} s_{1} \\
\quad+\sum_{n=1}^{\infty}(n+1)(n+2)(n+3) c_{n+3} \varsigma^{n}+6 c_{3}=0 .
\end{array}\right.
$$

Through comparing the coefficients of $\varsigma$, we can easily get the following results.

where $n=0$,

$$
s_{1}=-1-c_{0} c_{1}, c_{3}=-\frac{c_{1}+s_{0} c_{1}+c_{0} s_{1}}{6},
$$

where $n \geq 1$,

$$
\begin{aligned}
s_{n+1}= & -\frac{1}{(n+1)} \sum_{k=0}^{n}(n+1-k) c_{k} c_{n+1-k}, \\
c_{n+3}= & -\frac{1}{(n+1)(n+2)(n+3)}\left[(n+1) c_{n+1}+\sum_{k=0}^{n}(n+1-k) s_{k} c_{n+1-k}\right. \\
& \left.+\sum_{k=0}^{n}(n+1-k) c_{k} s_{n+1-k}\right] .
\end{aligned}
$$

Accordingly, the power series solution of the reduction Equation (C) is as follows

$$
\begin{aligned}
F(\varsigma)= & c_{0}+c_{1} \varsigma+c_{2} \varsigma^{2}-\frac{c_{1}+s_{0} c_{1}+c_{0} s_{1}}{6} \varsigma^{3} \\
& -\frac{1}{(n+1)(n+2)(n+3)} \sum_{n=1}^{\infty}\left[(n+1) c_{n+1}+\sum_{k=0}^{n}(n+1-k) s_{k} c_{n+1-k}\right. \\
& \left.+\sum_{k=0}^{n}(n+1-k) c_{k} s_{n+1-k}\right] \varsigma^{n+3}, \\
H(\varsigma)= & s_{0}-\left(1+c_{0} c_{1}\right) \varsigma-\frac{1}{(n+1)} \sum_{n=1}^{\infty} \sum_{k=0}^{n}(n+1-k) c_{k} c_{n+1-k} \varsigma^{n+1} .
\end{aligned}
$$

And then we get the following power series solution of Equation (1)

$$
\begin{aligned}
u(x, t)= & t+c_{0}+c_{1}\left(\frac{-t^{2}+2 x}{2}\right)+c_{2}\left(\frac{-t^{2}+2 x}{2}\right)^{2} \\
& -\frac{c_{1}+s_{0} c_{1}+c_{0} s_{1}}{6}\left(\frac{-t^{2}+2 x}{2}\right)^{3} \\
& -\frac{1}{(n+1)(n+2)(n+3)} \sum_{n=1}^{\infty}\left[(n+1) c_{n+1}+\sum_{k=0}^{n}(n+1-k) s_{k} c_{n+1-k}\right. \\
& \left.+\sum_{k=0}^{n}(n+1-k) c_{k} s_{n+1-k}\right]\left(\frac{-t^{2}+2 x}{2}\right)^{n+3},
\end{aligned}
$$




$$
\begin{aligned}
v(x, t)= & s_{0}-\left(1+c_{0} c_{1}\right)\left(\frac{-t^{2}+2 x}{2}\right) \\
& -\frac{1}{(n+1)} \sum_{n=1}^{\infty} \sum_{k=0}^{n}(n+1-k) c_{k} c_{n+1-k}\left(\frac{-t^{2}+2 x}{2}\right)^{n+1} .
\end{aligned}
$$

\subsection{Explicit Solutions of the Reduction Equation (D) Using Extended Tanh Function Method}

Using extended tanh function method, similar to the solving of the reduction Equation (B), we obtain the following results:

$$
\begin{aligned}
& a_{0}= \pm \rho, a_{1}= \pm 2 \omega, A_{1}= \pm 2 \lambda, b_{0}=-1, b_{1}=-2 \rho \omega, \\
& b_{2}=-2 \omega^{2}, B_{1}=-2 \lambda \rho, B_{2}=-2 \lambda^{2} .
\end{aligned}
$$

and selecting the following hyperbolic function

$$
\phi=-\frac{1}{2 \omega}\left(\rho+\sqrt{\theta} \operatorname{coth}\left[\frac{\sqrt{\theta}}{2} \varsigma\right]\right)
$$

We obtain explicit solutions of the Equation (1.1) as follow

$$
\left\{\begin{aligned}
& u(x, t)= \mp \sqrt{\theta} \operatorname{coth}\left[\frac{\sqrt{\theta} \varsigma}{2}\right] \mp \frac{4 \lambda \omega}{\rho-\sqrt{\theta} \operatorname{coth}\left[\frac{\sqrt{\theta} \varsigma}{2}\right]}, \\
& v(x, t)=-1+\frac{\rho^{2}}{2}-\frac{1}{2} \theta \operatorname{coth}^{2}\left[\frac{\sqrt{\theta} \varsigma}{2}\right]-\frac{8 \lambda^{2} \omega^{2}}{\left(-\rho+\sqrt{\theta} \operatorname{coth}\left[\frac{\sqrt{\theta} \varsigma}{2}\right]\right)^{2}} \\
&+\frac{4 \lambda \rho \omega}{\rho+\sqrt{\theta} \operatorname{coth}\left[\frac{\sqrt{\theta} \varsigma}{2}\right]},
\end{aligned}\right.
$$

where $\varsigma=x-t, \theta=\rho^{2}-4 \lambda \omega<0 \quad$ (see Figure 2).

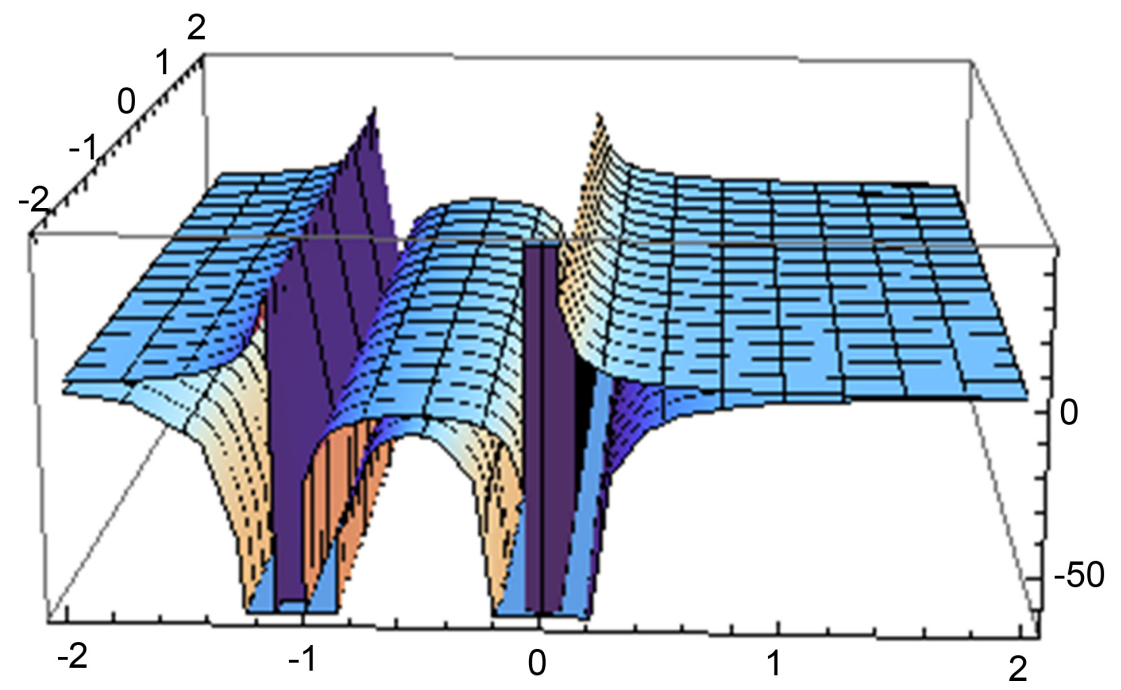

Figure 2. Plot of explicit solution above with $\lambda=4, \rho=2, \omega=0.2$. 


\section{Conclusion}

In the field of physics and engineering mechanics, it is particularly important to solve nonlinear differential equations. In the work, the Lie group analysis method has been employed to investigate the dispersive long wave equations. Based on this method, the vector fields and symmetry reductions have been obtained for the system. Since it is difficult to solve the reduction equations directly, the power series method and the extended Tanh function method have been used to construct more explicit solutions, which can enrich the exact solutions of the dispersive long wave equations. The basic idea is efficient and powerful in solving wide classes of nonlinear differential equations.

\section{Acknowledgements}

The authors would like to express their thanks to the unknown referees for their careful reading and helpful comments. Project supported by the Natural Science Foundation of Inner Mongolia, China (Grant No. 2016MS0116).

\section{Conflicts of Interest}

The authors declare no conflicts of interest regarding the publication of this paper.

\section{References}

[1] Marin, M. (1997) On Weak Solutions in Elasticity of Dipolar Bodies with Voids. Journal of Computational and Applied Mathematics, 82, 291-297. https://doi.org/10.1016/S0377-0427(97)00047-2

[2] Adomian, G. (1998) A Review of the Decomposition Method in Applied Mathematics. Journal of Computational and Applied Mathematics, 135, 501-544.

[3] Fan, E.G. (2003) Uniformly Constructing a Series of Explicit Exact Solutions to Nonlinear Equations in Mathematical Physics. Chaos, Solitons \& Fractals, 16, 819-839. https://doi.org/10.1016/S0960-0779(02)00472-1

[4] Broadbridge, P., Moitsheki, R.J. and Edwards, M.P. (2002) Analytical Solutions for Two Dimensional Solute Transport with Velocity-Dependent Dispersion. Environmental Mechanics, 129, 145-153. https://doi.org/10.1029/129GM14

[5] Abdul-Majid, W. and Alice, G. (2004) An Analytic Study of Fishers Equation by using Adomian Decomposition Method. Applied Mathematics and Computation, 154,609-620. https://doi.org/10.1016/S0096-3003(03)00738-0

[6] Rogers, C. and Schief, W.K. (2002) Backlund and Darboux Transformations Geometry and Modern Applications in Soliton Theory. Cambridge University Press, Cambridge. https://doi.org/10.1017/CBO9780511606359

[7] Hirota, R. (2004) Direct Methods in Soliton Theory. Springer, Berlin. https://doi.org/10.1017/CBO9780511543043

[8] Ma, W.X. and Fan, E.G. (2011) Linear Superposition Principle Applying to Hirota Bilinear Equations Computers and Mathematics with Applications, 61, 950-959. https://doi.org/10.1016/j.camwa.2010.12.043

[9] Ma, W.X. (2015) Lump Solutions to the Kadomtsev-Petviashvili Equation. Physics Letters A, 379, 1975-1978. https://doi.org/10.1016/j.physleta.2015.06.061 
[10] Olver, P.J. (1986) Applications of Lie Groups to Differential Equations. Springer, New York. https://doi.org/10.1007/978-1-4684-0274-2

[11] Bluman, G.W. and Kumei, S. (1989) Symmetries and Differential Equations. Springer, Berlin. https://doi.org/10.1007/978-1-4757-4307-4

[12] Khalique, C.M. and Adem, K.R. (2011) Exact Solutions of the (2+1)-Dimensional Zakharov-Kuznetsov Modified Equal Width Equation Using Lie Group Analysis. Mathematical and Computer Modelling, 54, 184-189. https://doi.org/10.1016/j.mcm.2011.01.049

[13] Liu, H. and Li, J. (2009) Lie Symmetry Analysis and Exact Solutions for the Short Pulse Equation. Nonlinear Analysis. Theory, Methods Applications, 71, 2126-2133. https://doi.org/10.1016/j.na.2009.01.075

[14] Rosa, M., Bruzon, M.S. and Gandarias, M.L. (2015) Symmetry Analysis and Exact Solutions for a Generalized Fisher Equation in Cylindrical Coordinates. Communications in Nonlinear Science and Numerical Simulation, 25, 74-83. https://doi.org/10.1016/j.cnsns.2015.01.010

[15] Cantwell, B.J. (2002) Introduction to Symmetry Analysis. Cambridge University Press, Cambridge.

[16] Moussa, M.H.M. and Shikh, R.M.E. (2006) Similarity Reduction and Similarity Solutions of Zabolotskay-Khoklov Equation with Dissipative Term via Symmetry Method. Physica A: Statistical Mechanics and Its Applications, 371, 325-335. https://doi.org/10.1016/j.physa.2006.04.044

[17] Lukashchuk, S.Y. and Saburova, R.D. (2018) Approximate Symmetry Group Classification for A Nonlinear Fractional Filtration Equation of Diffusion-Wave Type. Nonlinear Dynamics, 93, 295-305. https://doi.org/10.1007/s11071-018-4192-3

[18] Liu, Y.K. and Li, B. (2016) Nonlocal Symmetry and Exact Solutions of the $(2+1)$ dimensional Gardner Equation. Chinese Journal of Physics, 54, 718-723. https://doi.org/10.1016/j.cjph.2016.05.014

[19] Qin, C.Y., Tian, S.F., Wang, X.B., Zou, L. and Zhang, T.T. (2018) Lie Symmetry Analysis, Conservation Laws and Analytic Solutions of the Time Fractional Kolmogorov-Petrovskii-Piskunov Equation. Chinese Journal of Physics, 56, 1734-1742. https://doi.org/10.1016/j.cjph.2018.05.002

[20] Bruzon, M.S. and Gandarias, M.L. (2011) Classical and Nonclassical Symmetries for the Krichever Novikov Equation. Theoretical and Mathematical Physics, 168, 875-885. https://doi.org/10.1007/s11232-011-0071-5

[21] Kudryashov, N.A. (2005) Simplest Equation Method to Look for Exact Solutions of Nonlinear Differential Equation. Chaos, Solitons \& Fractals, 24, 1217-1231. https://doi.org/10.1016/j.chaos.2004.09.109

[22] Xie, F.D., Chen, J. and Lu, Z.S. (2005) Using Symbolic Computation to Exactly Solve the Integrable Broer-Kaup Equations in (2+1)-Dimensional Space. Communications in Theoretical Physics, 43, 585-590. https://doi.org/10.1088/0253-6102/43/4/003

[23] He, J.H. (2011) Homotopy Perturbation Method for Bifurcation of Nonlinear Problems. International Journal of Nonlinear Sciences and Numerical Simulation, 6, 207-208.

[24] Barrio, R. (2005) Performance of the Taylor Series Method for ODEs/DAEs. Applied Mathematics and Computation, 163, 525-545.

https://doi.org/10.1016/j.amc.2004.02.015

[25] Yu, J.L., Li, F.Z. and She, L.B. (2017) Lie Symmetry Reductions and Exact Solutions 
of a Multidimensional Double Dispersion Equation. Applied Mathematics, 8, 712-723. https://doi.org/10.4236/am.2017.85056

[26] Feng, L.L., Tian S.F., Zhang, T.T. and Zhou, J. (2017) Lie Symmetries, Conservation Laws and Analytical Solutions for Two-Component Integrable Equations. Chinese Journal of Physics, 55, 996-1010. https://doi.org/10.1016/j.cjph.2017.03.008

[27] Baleanua, D., Incc, M., Yusuf, A. and Aliyuc, A.I. (2018) Lie Symmetry Analysis, Exact Solutions and Conservation Laws for the Time Fractional Caudrey-Dodd-Gibbon-Sawada-Kotera Equation. Communications in Nonlinear Science and Numerical Simulation, 59, 222-223. https://doi.org/10.1016/j.cnsns.2017.11.015

[28] Broer, L.J.F. (1975) Approximate Equations for Long Water Waves. Applied Scientific Research, 31, 377-395. https://doi.org/10.1007/BF00418048

[29] Kupershmidt, B.A. (1985) Mathematics of Dispersive Water Waves. Communications in Mathematical Physics, 99, 51-73. https://doi.org/10.1007/BF01466593

[30] Boiti, M., Leon, J.J.P. and Pempinelli, F. (1987) Spectral Transform for a Two Spatial Dimension Extension of the Dispersive Long Wave Equation. Inverse Problems, 3, 371-387. https://doi.org/10.1088/0266-5611/3/3/007

[31] Eckhaus, W. (1985) Department of Mathematics. University of Utrecht, Utrecht.

[32] Zhang, J.F., Guo, G.P. and Wu, F.M. (2002) New Multi-Soliton Solutions and Travelling Wave Solutions of the Dispersive Long-Wave Equations. Chinese Physics, 11, 533-536. https://doi.org/10.1088/1009-1963/11/6/303

[33] Zayed, E.M.E. and Alurrfi, K.A.E. (2013) The Modified Extended Tanh Function Method and Its Applications to the Generalized KdV-mKdV Equation with Any-Order Nonlinear Terms. International Journal of Environmental Engineering Science and Technology Research, 1, 165-170. 